\title{
POTENCIAL PRODUTIVO DE UMA PASTAGEM NATIVA DO RIO GRANDE DO SUL SUBMETIDA A NÍVEIS DE OFERTA DE FORRAGEM ${ }^{1}$
}

\author{
POTENTIAL PRODUCTION OF A SOUTHERN BRAZIL RANGELAND SUBMITTED \\ TO FORAGE ON OFFER LEVELS
}

\author{
Eduardo Londero Moojen ${ }^{2}$ Gerzy Ernesto Maraschin ${ }^{3}$
}

RESUMO

Um experimento de pastejo em pastagem nativa foi conduzido na Estação Experimental Agronômica, UFRGS, Eldorado do Sul, RS, para avaliar o ganho médio diário, o ganho por hectare de novilhos de corte, taxa de acumulação de massa seca da pastagem, produção de matéria seca, conteúdo de proteína bruta, digestibilidade in vitro da matéria orgânica e percentagem de material morto na matéria seca, sob níveis de $4,0,8,0,12,0$ e $16,0 \%$ do peso vivo de oferta de forragem. As ofertas de forragem condicionaram diferentes resíduos de massa seca, e moldaram diferentes perfis. A redução na pressão de pastejo provocou aumento no resíduo. Diferentes quantidades de forragem em oferta afetaram a resposta animal. A melhor taxa de acumulação de massa seca da pastagem, ganho médio diário e ganho por hectare foram obtidos na faixa de 11,5 a $13,4 \%$ de oferta de forragem. A qualidade da forragem for reduzida com o aumento da quantidade de forragem em oferta.

Palavras-chave: oferta de forragem, pressão de pastejo, resíduos de MS, ganho médio diário, ganho de peso vivo por hectare, produção animal.

\section{SUMMARY}

A grazing experiment on rangeland was conducted at the Estação Experimental Agronômica, UFRGS, Eldorado do Sul, RS, Brazil, to evaluate the average daily gain per animal and liveweight gain per hectare of beef cattle, pasture accumulation rate, dry-matter production, crude protein content, in vitro organic matter digestibility and litter percentage in dry matter, under levels of 4.0, 8.0, 12.0 and 16.0\% liveweight as forage on offer. Forage on offer conditionated different residual dry matter/area. By reducing the grazing pressue there was an increase in residual dry matter/ha. Different amounts of forage on offer affected animal performance. The best accumulation rate of the pasture, average daily gain and liveweigh gain/ha were obtained under the range of 11.5 and $13.4 \%$ of forage on offer. The quality of dry matter available was reduced by increasing the amount of forage on offer.

Key words: forage on offer, grazing pressure, residual DM, average daily gain per animal, liveweigh gain per hectare, animal production.

\section{INTRODUÇÃO}

As pastagens naturais cobrem uma área aproximada de 12 milhões de hectares e constituem o grande recurso forrageiro responsável pela produção bovina e ovina no Rio Grande do Sul. Este recurso forrageiro vem sendo utilizado desde o início do século 17, quando foi introduzido o gado bovino na região Sul, e vem sendo mal explorado sem uma base conhecida. Há, portanto, uma necessidade de se buscar em formas de utilização mais racionais deste recurso forrageiro e de integrálo nos diferentes sistemas de produção agropecuários com alternativas que possam complementá-lo, como o uso de pastagens cultivadas, a conservação de forragem nas suas diferentes formas, o próprio melhoramento desta pastagem natural pela introdução de espécies, adubação, etc.

A utilização das pastagens naturais é ditada por um conceito bastante conhecido, a sua lotação, como expressão de carga. São usadas, normalmente, taxas um tanto empíricas. Um

\footnotetext{
${ }^{1}$ Parte da Tese de Doutorado apresentada pelo primeiro autor ao Curso de Pós-graduação em Zootecnia, Universidade Federal do Rio Grande do Sul (UFRGS).

${ }^{2}$ Engenheiro Agrônomo, Doutor, Professor Titular, Departamento de Zootecnia, Universidade Federal de Santa Maria, 97105-100, Santa Maria, RS. E-mail: moojenel @ ccr.ufsm.br. Autor para correspondência.

${ }^{3}$ Engenheiro Agrônomo, PhD., Professor Adjunto, Departamento de Plantas Forrageiras e Agrometeorologia, UFRGS, Porto Alegre, RS, Pesquisador aposentado do CNPq.
} 
conceito mais apropriado seria a pressão de pastejo (PP), que MOTT (1960) definiu como o número de animais por unidade de forragem disponível, expressa habitualmente em base percentual e diária do peso vivo dos animais, distintamente de lotação, que é definida como o número de animais por unidade de área. Conforme o mesmo autor, taxa de lotação não apresenta relação alguma com a disponibilidade de forragem No entanto, parece que oferta de forragem (OF) é mais condizente com nossas condições ambientais.

A imposição de diferentes PP a uma pastagem, afeta a qualidade, produção de massa seca (MS), composição botânica e persistência da pastagem, e com isto condiciona diferentes ganhos por animal e ganhos por área (BRYANT et al., 1970). Diferentes PP condicionam diferentes quantidades de MS/ha ou resíduo de MS aérea. Com a redução na PP tem-se maiores resíduos (BURNS et al., 1984; ESCOSTEGUY, 1990; MORAES, 1991). Esta condição afeta a taxa de crescimento da pastagem e sua conseqüente produção de MS (KORTE et al., 1987).

ESCOSTEGUY (1990), em pastagem natural submetida a OF de $4,0,8,0,12,0$ e 16,0\% quantificou, respectivamente, resíduos de 486, 847, 1421 e $1785 \mathrm{~kg}$ de $\mathrm{MS} / \mathrm{ha}$. As taxas de crescimento da pastagem foram crescentes com o aumento das OF até $12 \% \mathrm{PV}$, sendo de 15,$3 ; 21,5 ; 24,4$ e $21,3 \mathrm{~kg}$ de MS/ha /dia. A qualidade da MS, medida pelo conteúdo de $\mathrm{PB}$ e digestibilidade in vitro da matéria orgânica (DIVMO) diminuiu com o aumento do resíduo (ESCOSTEGUY, 1990). Uma relação linear entre a porcentagem de PB foi observada, com valores de \% de $\mathrm{PB}$ de 7,8, 6,7, 5,7 e 5,5, respectivamente, para as $\mathrm{OF}$ de 4,0, 8,0, 12,0 e $16,0 \%$ (ESCOSTEGUY, 1990). No entanto, os teores de PB e digestibilidade da forragem em oferta tem valor relativo, na medida em que o animal em pastejo, quando há oportunidade de exercer a seletividade, pode obter uma dieta de qualidade superior à oferecida (STOBBS, 1973; 1975).

As diferentes ofertas de MS proporcionadas pelas PP afetam o consumo. A relação entre o consumo e a quantidade de forragem disponível, segundo POPPI et al. (1987) é curvilinear assintótica. Conforme HODGSON et al. (1971), a taxa de ganho de peso vivo é reduzida quando a quantidade de forragem deixada após o pastejo cai abaixo de 2000 a $2500 \mathrm{~kg} / \mathrm{ha}$ de matéria orgânica. WILLOUGHBY (1958) observou que os ganhos de peso vivo em ovinos eram limitados quando a disponibilidade de forragem era inferior à faixa de $1200-1600 \mathrm{~kg}$ de MS/ha. GIBB \& TREACHER (1976) observaram que se a disponibilidade de forragem não for, no mínimo, três vezes superior ao consumo diário de cordeiros, o mesmo pode ser restringido.

A relação entre ganho médio diário e PP conforme proposta por JONES \& SANDLAND (1974) seria linear, enquanto MOTT \& MOORE (1985) evidenciam que esta relação é quadrática ou curvilinear, e o ganho por área, dentro de amplos limites de PP, apresenta uma resposta quadrática.

O objetivo deste trabalho foi avaliar a resposta da pastagem nativa submetida a diferentes níveis de oferta de forragem.

\section{MATERIAL E MÉTODOS}

O experimento foi conduzido em uma área de campo nativo, na Estação Experimental Agronômica da UFRGS, em Eldorado do Sul, RS, de setembro de 1989 a abril de 1990 (224 dias). Os tratamentos aplicados foram níveis de oferta de forragem de 4,0, 8,0, 12,0 e 16,0kg de MS por $100 \mathrm{~kg}$ de peso vivo por dia.

$\mathrm{O}$ experimento foi em arranjo fatorial $4 \times 4 \times 5$ (4 ofertas de forragem $x 4$ épocas de diferimento x 5 níveis de adubação) com um delineamento em parcelas sub-sub-divididas. As parcelas principais foram arranjadas em blocos com duas repetições e a finalidade do bloqueamento foi o controle da variação do terreno. As sub-parcelas foram designadas ao acaso dentro das parcelas principais e as sub-sub-parcelas idem dentro daquelas. Cada parcela principal foi constituída por um potreiro com área variando de 3,05 a 10,06ha.

A metodologia estatística inclui o método de variância, teste $\mathrm{F}$ e ajuste de equações de regressão polinomial.

Foi usado o pacote estatístico SAS (SAS, 1993) em computador IBM 9276 do Centro de Processamento de Dados da UFSM.

As amostragens para estimar a MS/ha seguiram o método de dupla amostragem (WILM et al., 1944), conjugando em cada avaliação e em cada potreiro dez amostras de corte (cinco em áreas protegidas e cinco em áreas acessíveis ao pastejo), e um número variável de leituras aleatórias de um disco calibrado, em transectas pré-definidas. Os cortes foram feitos com tesouras de esquilar, ao nível do solo. Nos potreiros com oferta de forragem de 4,0\% foram tomadas 20 leituras de disco em cada um, 40 leituras nos de 8,0\%, e 60 leituras nos de 12,0 e $16,0 \%$, totalizando 360 leituras de disco.

As amostras de corte foram secas em estufa com ar forçado a $60^{\circ} \mathrm{C}$, para estimar a MS. Destas, as amostras das áreas acessíveis ao pastejo, de cada potreiro, formaram amostras compostas por OF que foram moídas para análises posteriores. As 
amostragens para a estimativa da produção de MS e da taxa de acumulação de MS seguiram a técnica proposta por KLINGMAN et al. (1943) (ESCOSTEGUY, 1990). Usaram-se as mesmas áreas protegidas e acessíveis ao pastejo para estas estimativas. Foram feitas amostragens para a estimativa do material morto na MS, com a mesma freqüência das amostragens anteriores, cortando-se rente ao solo com tesoura de esquilar 15 subamostras aleatórias por potreiro, que compuseram amostras compostas para separação manual de material vivo e morto na MS.

O sistema de pastejo foi o de lotação contínua com carga variável, e para tanto, foi utilizada a técnica do uso de animais reguladores, descrita por MOTT \& LUCAS (1952). Utilizaram-se quatro novilhos testadores por potreiro, e um número variável de animais reguladores, para manter os níveis de $\mathrm{OF}$ desejados. O ajuste da carga animal foi feito baseado na estimativa da MS somada à taxa de acumulação estimada no período imediatamente anterior, que era projetada para o período seguinte. Esta soma resultou na estimativa da disponibilidade de MS dos potreiros. Com intervalos de cerca de 28 dias foram feitas pesagens dos animais, após um jejum prévio de 12 horas, e os ajustes de carga para manter as ofertas pretendidas.

Para cada intervalo entre avaliações, e na média final, calculou-se a OF real, ou seja, a OF efetivamente praticada, substituindo-se a taxa de acumulação projetada pela estimada no período. Neste estudo, optou-se pela relação da OF real, e não da pretendida, com as demais variáveis.

Os ganhos médios diários (GMD) foram obtidos periodicamente subtraindo o peso médio final dos novilhos testadores do seu peso médio inicial, dividindo pelo período de tempo transcorrido. O cálculo da capacidade de suporte foi baseado no método de PETERSEN \& LUCAS (1968), e a expressão da carga foi feita em animais.dia/ha.

$\mathrm{O}$ teor de nitrogênio $(\mathrm{N})$ da forragem produzida foi determinado pelo método semi-micro Kjeldhal, descrito por BREMNER (1965). A porcentagem de $\mathrm{N}$ da MS foi multiplicada por 6,25 para estimar a porcentagem de proteína bruta das amostras. A digestibilidade in vitro da matéria orgânica foi determinada pelo método de TILLEY \& TERRY (1963).

\section{RESULTADOS E DISCUSSÃO}

A relação entre a MS/ha média e a oferta foi melhor expressa por uma regressão linear $(\mathrm{P}<$ $0,0005)$, como se observa na figura 1 . A redução na PP (aumento de oferta) levou a uma condição de maior MS/ha.

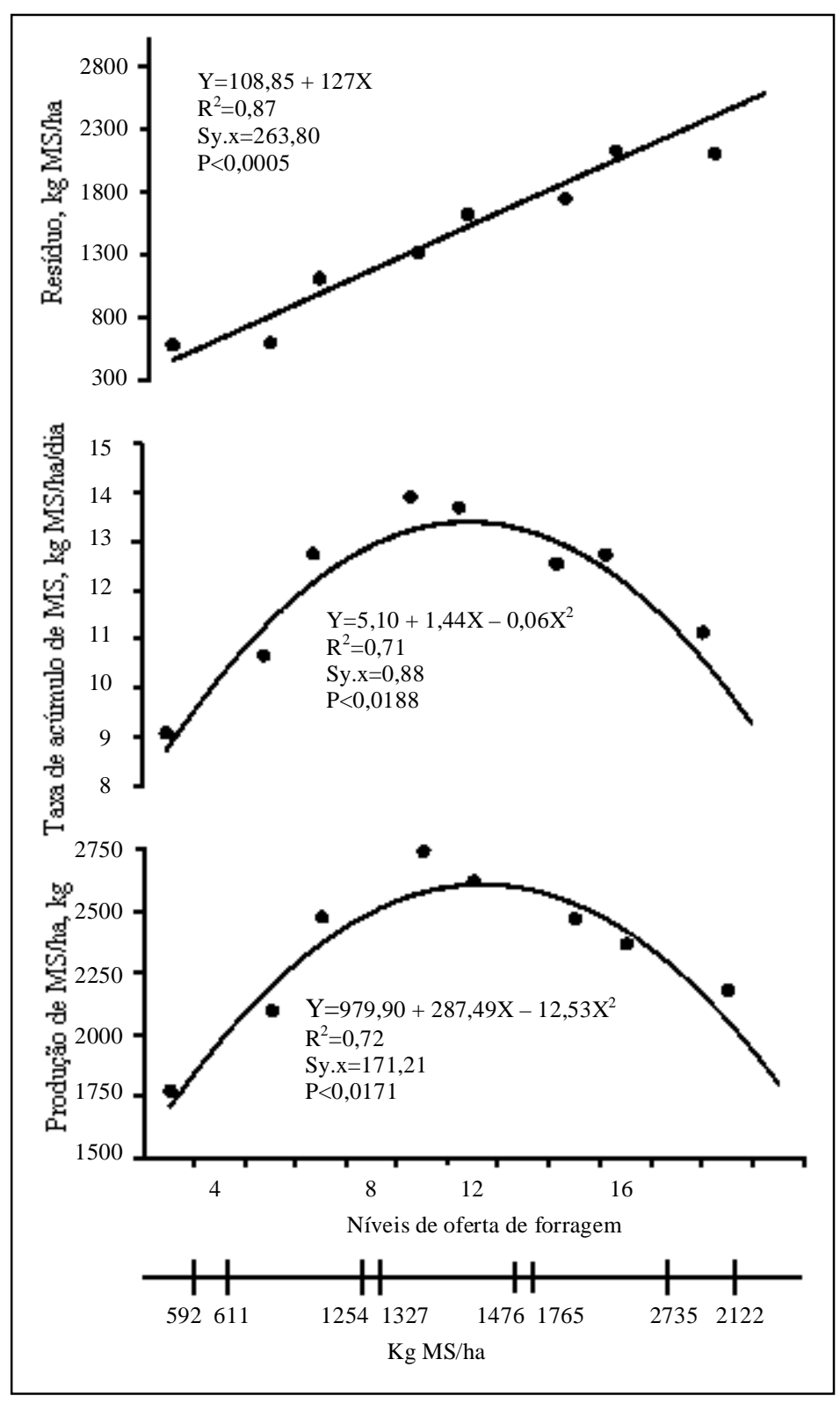

Figura 1 - Resíduos kg MS/ha), taxa de acumulação de MS (kg MS/ha/dia) e produção de MS/ha $(\mathrm{kg})$ do campo nativo submetido a níveis de oferta de forragem. EEA,UFRGS, Eldorado do Sul. 
A taxa de acumulação de MS da pastagem nas diferentes ofertas também é apresentada na figura 1. A relação entre estas variáveis foi melhor explicada pela regressão quadrática $(\mathrm{P}<0,0188)$. Com o aumento da OF, ocorreu um aumento na taxa de acumulação até um máximo, seguida de uma redução na taxa de acumulação, com novos aumentos na oferta. A máxima taxa de acumulação de MS da pastagem correspondeu a uma oferta de $11,5 \%$ PV. Resposta semelhante deste parâmetro foi obtida por MARASCHIN \& MOTT (1989). ESCOSTEGUY (1990) também obteve uma resposta semelhante a esta em pastagem nativa, observando a maior taxa de acumulação de MS na OF de $12,0 \% \mathrm{PV}$, quando trabalhou com OF de 3 a $12 \%$. No segundo ano, trabalhando com OF de 4 a $16 \%$, observou melhores respostas nas OF entre 8 a $12 \%$.

A produção de MS da pastagem sob o efeito de oferta é apresentada na figura 1. A relação entre estas variáveis foi quadrática $(\mathrm{P}<0,0171)$, o que é lógico, quando se sabe que a produção de MS é a expressão da taxa de acumulação de MS diária pelo tempo transcorrido.

A porcentagem de $\mathrm{PB}$ em função das diferentes ofertas é mostrada na figura 2 , na qual pode ser observado que, com o aumento da OF, houve decréscimo linear $(\mathrm{P}<0,004)$ na porcentagem de $\mathrm{PB}$. BOGDAN (1977) afirma que ocorre redução na porcentagem de $\mathrm{PB}$ com $\mathrm{o}$ avanço no estádio de desenvolvimento das plantas forrageiras. Com o aumento da MS/ha, ou resíduo após pastejo, ESCOSTEGUY (1990)obteve uma resposta linear decrescente.

Os resultados da DIVMO do campo nativo, em função da imposição de diferentes ofertas, são apresentados na figura 2. Dos modelos testados, a regressão linear apresentou o melhor ajuste entre as variáveis $(\mathrm{P}<0,0454)$. Com a redução na $\mathrm{PP}$, maior $\mathrm{OF}$, ocoreu uma redução linear na DIVMO. A digestibilidade da forragem é reduzida pelo avanço no estádio de desenvolvimento das plantas (HACKER \& MINSON, 1981). Segundo HACKER \& MINSON (1981), isto ocorre pelo aumento na quantidade de componentes da parede celular e pela lignificação.

O envelhecimento da forragem disponível foi quantificado em termos de porcentagem de material morto $(\mathrm{MM})$ na $\mathrm{MS}$. A regressão linear $(\mathrm{P}<0,0006)$ apresentou $\mathrm{o}$ melhor ajuste entre as

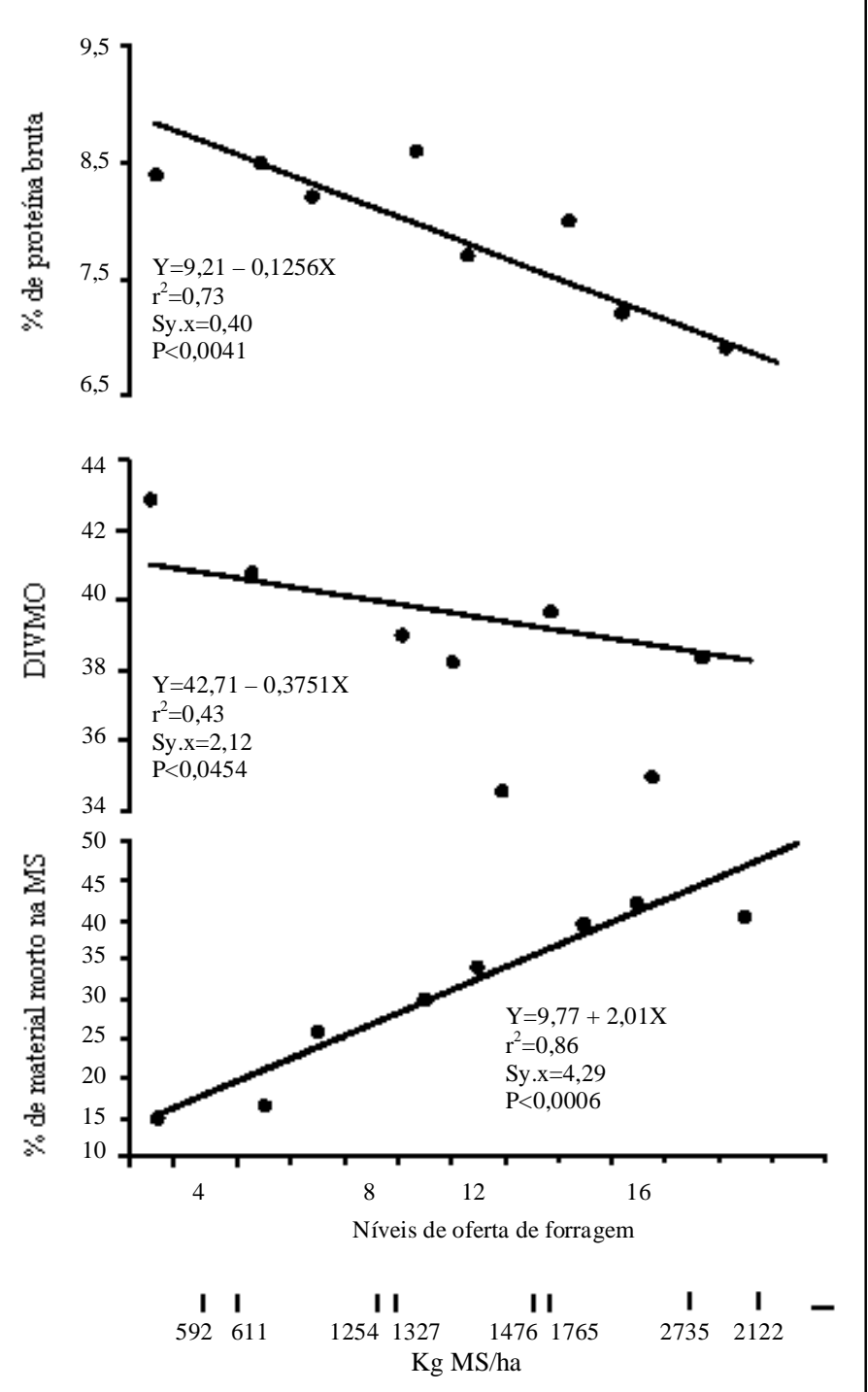

Figura 2 - Percentagem de proteína bruta média, digestibilidade da matéria orgânica \% e porcentagem de material morto do campo nativo submetido a níveis de oferta de forragem. EEA, UFRGS, Eldorado do Sul.

varáveis MM e PP, como se observa na figura 2. Assim, com a maior OF, ocorreu um aumento na porcentagem de MM na MS da forragem. Ao longo da estação de pastejo observou-se uma redução na porcentagem média de MM dos tratamentos, de onde se pode inferir que do início do outono ao final do inverno, o MM torna a aumentar sua participação na MS disponível.

O GMD nas diferentes ofertas de forragem é apresentado na figura 3. A regressão quadrática ( $\mathrm{P}<0,0815)$ expressou um bom ajuste entre as variáveis no nível referido. O GMD máximo foi de $0,540 \mathrm{~kg}$ na oferta de $13,4 \%$. Esta resposta coincide com MOTT \& MOORE (1985) que 


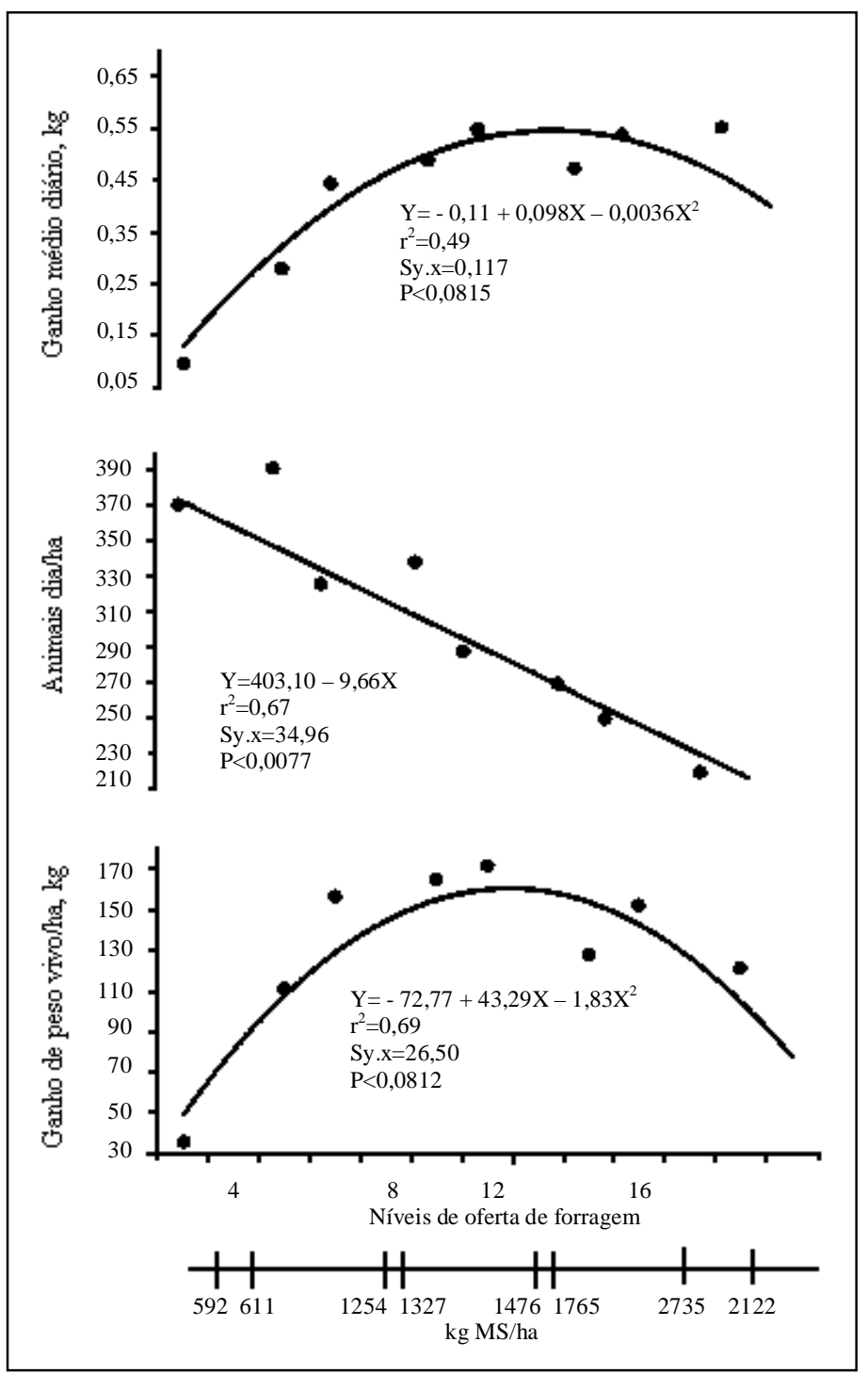

Figura 3 - Ganho médio diário $(\mathrm{kg})$, animais dia/ha $\left(\mathrm{n}^{\circ}\right)$ e ganho de peso vivo/ha $(\mathrm{kg})$ no campo nativo submetido a níveis de oferta de forragem. EEA, UFRGS, Eldorado do Sul.

sugerem ser esta relação quadrática ou curvilinear. Animais.dia/ha, como expressão de carga, e oferta de forragem se relacionaram por uma regressão linear $(\mathrm{P}<0,0077)$ (Figura 3). Com o aumento da OF, houve uma redução no número de animais na pastagem. Observa-se, pois, que, para manter uma pastagem mais folgada, é necessário reduzir a carga por unidade de área.

A relação entre o ganho/ha com a pressão de pastejo é apresentada na figura 3. Ressalte-se que foram descartados os valores de ganhos na PP de $12 \%$. A razão para tal foi que, neste tratamento, ocorreu um pastejo seletivo, ou seja, havia áreas efetivamente muito pastejadas e áreas praticamente descartadas. Assim, a PP efetiva nestes potreiros foi diferente e mais alta do que a PP real e PP pretendida. Testando diferentes modelos, sem os dados destes dois potreiros, observa-se na figura 3 , que a regressão quadrática $(\mathrm{P}<0,0812)$ apresentou o melhor ajuste para as variáveis, assemelhando-se ao modelo proposto por MOTT (1960). O ganho/ha máximo foi de $185 \mathrm{~kg}$ na oferta de forragem de $11,8 \%$.

\section{CONCLUSÕES}

A variação na pressão de pastejo condiciona diferentes quantidades de massa seca ou resíduo por área. Com a redução na pressão de pastejo, aumenta a MS/ha. Pelos ganhos obtidos por animal e por área, nas diferentes ofertas, estima-se que as ofertas de forragem em torno de $12,0 \%$ se situaram dentro da faixa, que correspondeu à melhor estimativa do potencial de produção de produto animal da pastagem.

\section{REFERÊNCIAS BIBLIOGRÁFICAS}

BOGDAN, A.V. Tropical pasture and fodder plants. New York : Longman, 1977. 475p.

BREMNER, J.M. Total nitrogen. In: BLACK, C.A. Methods of soil analysis. Madison : ASA, 1965. Pt.2, Chap.83, p.1149-1178. (Agronomy, 9).

BRYANT, H.T.; BLASER, R.E.; HAMMES JR., et al. Symposium on pasture methods for maximum production in beef cattle; effect of grazing management on animal an area output. J Anim Sci, Albany, v.30, n.1, p.153-158, 1970.

BURNS, J.C.; MOCHRIE, R.D.; TIMOTHY, D.H. Steer performance from two perennial Pennisetum species, switchgrass, and a fescue-Coastal bermudagrass system. Agron J, Madison, v.76, p.758-800, 1984.

ESCOSTEGUY, C.M.D. Avaliação agronômica de uma pastagem natural sob níveis de pressão de pastejo. Porto Alegre - RS, 1990. 231p. Dissertação (Mestrado em Fitotecnia) - Curso de Pós-graduação em Agronomia, Universidade Federal do Rio Grande do Sul, 1990.

GIBB, M.J.; TREACHER, T.T. The effect of herbage allowance on herbage intake and performance of lambs grazing perennial ryegrass and clover swards. J Agric Sci, Canberra, v.86, p.355-365, 1976.

HACKER, J.B.; MINSON, D.J. The digestibility of plant parts. Herb Abstr, Oxford, v.51, n.9, p.459-482, 1981.

HODGSON, J.; TAYLER, J.C.; LONSDALE, C.R. The relationship between intensity of grazing and the herbage consumption and growth of calves. J Br Grassind Soc, Aberystwyth, v.26, p.231-237, 1971. 
JONES, R.J.; SANDLAND, R.L. The relation between anima gain and stocking rate. J Agric Sci, Canberra, v.83, p.335342, 1974.

KORTE, C.J.; CHU, A.C.P.; FIELD, T.R.O. Pasture production. In: NICOL, A.M. Feeding livestock on pasture. Hamilton: N Z Soc Anim Prod., 1987 p.7-20. (Occasional publication, 10)

KLINGMAN, D.L.; MILES, S.R.; MOTT, G.O. The cage method for determining consumption and yield of pasture herbage. J Am Soc Agron, Geneva, v.35, p.739-746, 1943.

MORAES, A. de. Produtividade animal e dinâmica de uma pastagem de pangola (Digitaria decumbens Stent), azevém (Lolium multiflorum Lam.) e trevo branco (Trifolium repens L.), submetida a diferentes pressões de pastejo. Porto Alegre - RS, 1991. 200p. Tese (Doutorado em Zootecnia) - Curso de Pós-graduação em Zootecnia, Universidade Federal do Rio Grande do Sul, 1991.

MOTT, G.O.; LUCAS, H.L. The design, conduct and interpretation of grazing trials in cultivated and improved pastures. In: INT GRASSLND CONG, 6, 1952 Pennsylvania. Proceedings... Pennsylvania : State College, 1952. p.1380-1385.

MOTT, G.O. Grazing pressure and the measurement of pasture production. In: INT GRASSLND CONG, 8, 1960, Reading, England. Proceedings... Oxford : Alden, 1960. p.606-664.

MOTT, G.O.; MOORE, J.E. Evaluating forage production. In: HEATH, M.E., BARNES, R.F., METCALFE, D.S. Forages. 4 ed. Ames : Iowa State University, 1985. Chap. 45.
PETERSEN, R.G.; LUCAS, H.L. Computing methods for the evaluation of pastures by means of animal response. Agron J, Madison, v.60, n.6, p.682-687, 1968.

POPPI, D.P.; HUGHES, T.P.; L'HUILLIER, P.J. Intake of pasture by grazing ruminants. In: NICOL, A.M. Feeding livestock on pasture. Hamilton : N Z Soc An Prod., 1987, p.55-63. (Occasional publication, 10)

SAS Institute. STAT USER'S GUIDE. Version 6.08. Cary, 1993. 846p.

STOBBS, T.H. The effect of plant structure on the intake of tropical pasture. II. Differences in sward structure, nutritive value, and bite size of animals grazing Setaria anceps and Chloris gayana at various stages of growth. Aust J Agric Res, Melbourne, v.24, n.6, p.821-829, 1973.

STOBBS, T.H. The effect of plant structure of fertilizer nitrogen on the size of bite harvested by Jersey cows grazing Setaria anceps cv. Kazungula swards. Aust J Agric Res, Melbourne, v.26, p.997-1007, 1975

TILLEY, J.M.A.; TERRY, R.A. A two-stage technique for the in vitro digestion of forage crop. J Br Grasslnd Soc, Hurley, v.18, n.2, p.104-111, 1963.

WILLOUGHBY, W.W. The relationship of pasture availability and animal production. In: BIENAL CONFERENCE, 2, 1958, Canberra. Proceedings... Canberra : Aust Soc An Prod, 1958. v.2, p.42-45.

WILM, H.G.; COSTELlO, D.F.; KLIPPLE, G.E. Estimating forage yield by the double sampling method. J Am Soc Agr, New York, v.36, n.1, p.194-203, 1944. 